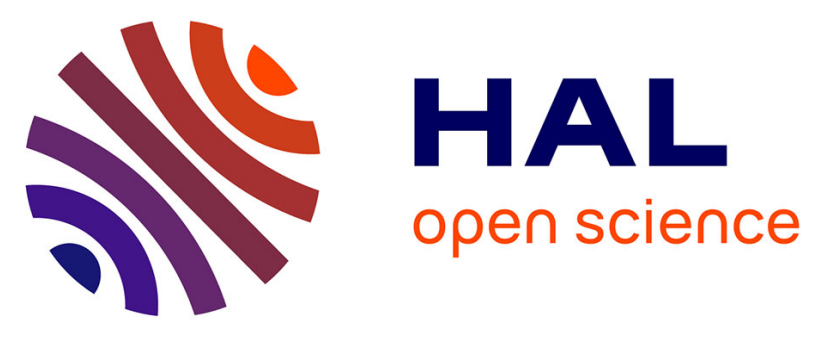

\title{
A year (2014-2015) of plants in Proteomics journal. Progress in wet and dry methodologies, moving from protein catalogs, and the view of classic plant biochemists
}

Rosa Sanchez-Lucas, Angela Mehta, Luis Valledor, Francisco Cabello Hurtado, M. Cristina Romero-Rodriguez, Lyudmila Simova Stoilova, Sekvan Demir, Luis E. Rodriguez De Francisco, Ana M. Maldonado Alconada, Ana L. Jorrin-Prieto, et al.

\section{To cite this version:}

Rosa Sanchez-Lucas, Angela Mehta, Luis Valledor, Francisco Cabello Hurtado, M. Cristina RomeroRodriguez, et al.. A year (2014-2015) of plants in Proteomics journal. Progress in wet and dry methodologies, moving from protein catalogs, and the view of classic plant biochemists. Proteomics, 2016, 16 (5), pp.866-876. 10.1002/pmic.201500351 . hal-01239816

\section{HAL Id: hal-01239816}

\section{https://hal-univ-rennes1.archives-ouvertes.fr/hal-01239816}

Submitted on 24 Mar 2016

HAL is a multi-disciplinary open access archive for the deposit and dissemination of scientific research documents, whether they are published or not. The documents may come from teaching and research institutions in France or abroad, or from public or private research centers.
L'archive ouverte pluridisciplinaire HAL, est destinée au dépôt et à la diffusion de documents scientifiques de niveau recherche, publiés ou non, émanant des établissements d'enseignement et de recherche français ou étrangers, des laboratoires publics ou privés. 
1 A year (2014-2015) of plants in Proteomics journal. Progress in wet and dry 2 methodologies, moving from protein catalogs, and the view of classic plant

\section{biochemists}

Rosa Sanchez-Lucas ${ }^{1}$, Angela Mehta ${ }^{2}$, Luis Valledor ${ }^{3}$, Francisco Cabello Hurtado ${ }^{4}$, M. Cristina Romero-Rodriguez ${ }^{5}$, Lyudmila Simova Stoilova ${ }^{6}$, Sekvan Demir ${ }^{1}$, Luis E. Rodriguez de Francisco ${ }^{1,7}$, Ana M Maldonado Alconada ${ }^{1}$, Ana L Jorrin-Prieto ${ }^{1}$, Jesus V. Jorrín-Novo ${ }^{1 *}$

${ }^{1}$ Agroforestry and Plant Biochemistry and Proteomics Research Group, Dpt. Biochemistry and Molecular Biology, University of Cordoba-CeiA3, Cordoba, Spain.

${ }^{2}$ Embrapa Recursos Genéticos e Biotecnologia (CENARGEN), Brasília, DF, Brazil.

${ }^{3}$ Dpt. Biology of Organisms and Systems (BOS), University of Oviedo, Oviedo, Spain.

${ }^{4}$ Mechanisms and Origin of Biodiversity Team, UMR 6553-Ecobio, University of Rennes, Rennes, France.

${ }^{5}$ Centro Multidisciplinario de Investigaciones Tecnológicas, and Dpto. de Fitoquímica, Facultad de Ciencias Químicas, Universidad Nacional de Asunción, San Lorenzo-Paraguay.

${ }^{6}$ Plant Molecular Biology Department, Institute of Plant Physiology and Genetics, Bulgarian Academy of Sciences, Sofia, Bulgaria.

${ }^{7}$ INTEC-Sto. Domingo, República Dominicana.

(*) Corresponding author

Author for correspondence. Jesus V. Jorrín Novo.

Bioquímica y Proteómica Vegetal y Agroforestal; Dpto. Bioquímica y Biología Molecular, Universidad de Córdoba.

Campus de Rabanales, Ed. Severo Ochoa, 14071 Córdoba, España

E-mail: bf1jonoj@uco.es; Phone +34 957 218574; FAX +34 957218439 
27 Key words: plant proteomics, proteogenomics, systems biology, signaling, signal transduction

29 Total number of words (including references as well as figure and table legends): 8164

30 Abstract

31 The present review is an update of the previous one published in Proteomics 2015 Reviews special 32 issue [1] covering the 2014-July 2015 period. It has been written on the bases of the publications that 33 appeared in Proteomics journal during that period and the most relevant ones that have been published 34 in other high-impact journals. Methodological advances and the contribution of the field to the 35 knowledge of plant biology processes and its translation to agroforestry and environmental sectors 36 will be discussed. This review has been organized in four blocks, with a starting general introduction 37 (literature survey) followed by sections focusing on the methodology (in vitro, in vivo, wet and dry), proteomics integration with other approaches (systems biology and proteogenomics), biological information, and knowledge (cell communication, receptors and signaling), ending with a brief mention of some other biological and translational topics to which proteomics has made some contribution. 


\section{INTRODUCTION}

The topic of plant proteomics was reviewed in 2015 on the basis of the articles published in Proteomics journal (from now just Proteomics, written in italics) between 2001, when the first issue appeared, and May 2014 [1]. The present review is an update covering the January 2014-July 2015 period in which methodological advances and the contribution of the field to the plant biology knowledge and its translation to agroforestry and environmental sectors will be discussed. It is not intended to be repetitive considering recent reviews but aiming to get to the reader for a deeper discussion of key topics. It was written to be included in the 2016 Proteomics Review Special Issue, since it is the last one of the series edited by Prof. Dunn, the editor in chief of Proteomics since it appeared and who recently retired. It is a matter of justice to recognize the enormous effort of Prof. Dunn in establishing and consolidating Proteomics as a leader journal as well as his scientific contribution to the field. The corresponding author, in particular, and the plant community, in general, should acknowledge the personal consideration and the support he always gave to us reflected, just as an example, in his input to the International Plant Proteomics Organization (INPPO; http://www.inppo.com/) society [2], even though plant studies are not within his field of investigation. $\mathrm{He}$, better than nobody else, has been an actor, director, and observer of the evolution of Proteomic Science [3].

According to PubMed, during the period of 2014-July 2015 Proteomics published 600 papers distributed in 40 issues, either regular or special including Reviews 2014 and 2015 Tissue Proteomics and Imaging Mass Spectrometry, Top-Down Proteomics, Focus on Biomedical Research Trends, Proteomics in Chromatin Biology and Epigenetics, Proteogenomics, Signal Transduction, Proteomics 
Proteomics, Views on Protein N-terminal Biology, and Protein Structure. These thematic issues

perfectly represented the most recent technical advances, the frontier of knowledge in biology, and the interest in translational biomedicine. Of 592 papers published in Proteomics, 133 were selected for preparing this review as they dealt with plants (33 original papers, 16 reviews, and 1 meeting report, representing around $10 \%$ of the total papers released by the journal) or presented the state of the art of the field from a biological, methodological or translational point of view.

After a PubMed search the terms proteomics or proteome in the title/abstract returned 8553 hits. Compared with the other -omics approaches, this figure was slightly or much higher than transcriptomics/transcriptome (7728) or metabolomics/metabolome (3064) searches, respectively. While looking for the combined use of the different omics approaches, PubMed provided 52 proteomics, transcriptomics, and metabolomics reports, as well as 113 proteomics and transcriptomics, and 56 proteomics and metabolomics papers. The integration of the different - omics approaches will be discussed in a further section.

In the surveyed period, plants continued to be underrepresented in the current proteomics literature, but significantly increasing, with 683 references in PubMed (almost 15\% of the total) while for humans, just as a comparative example, the figure was 2795 , with quite a few related to the study of cancer, as well as vascular and other diseases [4]. Despite the claimed general worry about food supply, environmental concerns, and climate change, these issues have not been properly reflected in research funding policy in most of the western countries and even less in developing nations. This issue is very well discussed by Donald Kennedy (President Emeritus at Stanford University, and a former editor-in-chief of Science) in Science's editorial "Building Agricultural Research" [5]. Human welfare depends on well-connected health and food supply [6-8]. Famine is the major cause of mortality on a worldwide basis, and increasing nutritional value of primary crops would drastically reduce health problems in the less developed countries [9]. The situation seems to be changing, but very slowly; at HUPO (https://www.hupo.org/) or general proteomics meetings such as European EuPA (http://www.eupa.org/) or Spanish SEProt (http://www2.cbm.uam.es/ seprot/), room for nonhuman proteomics and groups interested in different type of organisms (microorganisms, plant, and 
animals) has been recently created, or is under discussion. The vision or sensations at the plant specific organization INPPO is that we are surviving within the field as a scientific ghetto. With no doubt, plant and human research can and should benefit from each other. Plants provide an almost unlimited experimental system and despite being far in evolution they share an important number of genes [10], with similar mechanisms of, for example, immunity [11], and host-pathogen interactions [12]. In addition, plants may be sources of drugs for disease treatment [13] and also of allergens [14, 15]. Some more examples of common phenomena between plant and animals are totipotency, the property of the cells to be developmentally reprogrammed, the conceptual basis of the stem cell projects [16], and the involvement of telomeres and telomerases in senescence and aging [17].

The topic of plant proteomics continues to be extensively reviewed, reflecting its expansion, relevance, and expectations; although we should assume by now that there is a long distance between expectations and realities, and that the full potential of proteomics is far, once again if compared with humans, from being fully exploited in plant biology research and crop science [18-20]. Here, in Proteomics, and also in other journals, much attention has been given to proteomics of plant stresses responses, the major topic in plant biology because of its implication in crop protection and biotechnological improvement [21-23]. Up to 15 plant proteomics reviews have been recently published in Proteomics, most of them in the Vol. 15, Iss. 5-6 (Reviews 2015) and 10 (Plant-based Foods: Seed, Nutrition and Human Health). Strikingly, the thematic issues covering the most recent technical novelties or the frontier in proteomics and biology knowledge have none or at most two plant papers per issue; it was the case of Tissue Proteomics and Imaging Mass Spectrometry (Vol.14, Iss. 7-8, one plant reference by Maciel et al., 2014 [24]), and OMICS Views on Protein N-terminal Biology (Vol. 15, Iss. 4; two plant original papers by Zhang et al., 2015 [25] and Venne et al., 2015 [26]). The review by Walton et al. (2015) [27] on signaling, a topic later discussed, deserves special attention, and is one of the plant reviews we should not miss. Regarding plant reviews in other journals, at least 15 have appeared, with some of them covering topics that will be mentioned later, including software, database and repositories [28] PTMs [29, 30], and signaling [31]. 

and MIAPE were discussed in the context of proteomics work and publications. From his experience,

the failure to meet proper scientific standards and those requested for a proteomics paper is the most obvious and immediate reason for rejection. As just a simple example, the general terminology employed in the literature included terms such as protein expression, when proteomics approaches actually determine abundance $[1,32]$. Abundance is the result not only of gene expression (transcription and translation) but also of protein degradation and PTMs.

The list of referenced papers did not pretend to be exhaustive, but limited, according to the author's considerations, to those that presented some methodological, biological or translational novelties. Some of the revised papers fit in the general group of comparative analysis based on 2-DE coupled to MS analysis and they were not included. On the other hand, special attention was given to those using second to fourth generation techniques (labelling, gel-free, SRM/MRM [1]), those integrating classic and-omics approaches, or focused on PTMs and interactomics. Regarding the previous period reviewed, there have not been real novelties in the experimental (plant) system used, the biological process studied or the translational purpose pursued [1]. There is still a general need to go beyond the construction of a protein catalog accompanied by more speculation than real discussion, although better annotations and deeper knowledge is required in some cases.

\section{WET AND DRY METHODOLOGIES}

\section{In vitro and in situ proteomics analysis}

Proteomics is a discipline in expansion with continuous novelties and improvements in techniques, protocols, softwares and equipments $[33,34]$. This is also true for quite standard and well stablished protocols in the early proteomics workflow stages prior to MS analysis, as it is important to optimize them to each experimental system (plant, organ, tissue, cell or subcellular fraction). Thus, improved protocols dealing with protein extraction [35], depletion [36, 37], fractionation [38], gel 
staining [39], multiplexing [40], trypsin protein digestion [41] and sample preparation for shotgun analysis $[42,43]$ have recently been published.

The use of different MS platforms in plant proteomics analysis was previously discussed [1], however it is important to mention that new ion fragmentation procedures as alternative to the current collision induced dissociation, CID, and electron transfer dissociation, ETD, such as photodissociation, have been claimed to produce enhanced sequence coverage and higher confidence in sequence assignment $[44,45]$.

One of the limitations of proteomics, when compared to genomic and transcriptomic analyses, is that proteins cannot be amplified as can DNA and therefore, optimized and efficient extraction methods must be developed. Thus, the very laborious and time consuming used protocols and the number of replicates (the three biological replicates performed as a rule are not enough to perform solid statistical analysis and get confident biological conclusions [46], it is recommended to perform at least 5 biological replicates to allow a reliable estimation of residual variance when more than two treatments are compared) are the main bottleneck that makes proteomics unpopular among plant biologists. The use of an appropriate extraction method is even more relevant for PTMs or interactomics studies that request fractionation steps. These issues have been addressed by Krahmer et al (2015) [47], who compared different alternatives for sample preparation compatible with large numbers of samples in phosphoproteomics analysis.

Protein extraction is still challenging and constitutes a big difficulty in the analysis of specific proteins such as integral membrane proteins [48]. Integral membrane proteomics analyses involve complicated protocols that include membrane purification and protein extraction. In order to analyze the symbiosome integral membrane proteins, Clarke et al. (2015) [49] reported a protocol that included isolation of symbiosomes with three-step Percoll gradients and preparation of the membrane system by ultracentrifugation of the broken symbiosomes supernatant, followed by bicarbonate stripping and chloroform-methanol protein extraction prior to MS analysis. Although the excessive manipulation of the sample may interfere in the integral membrane protein analysis it has a great 
value from a descriptive point of view. Once the protein is identified, the expression of the corresponding genes and the associated phenotype can be assessed by using complementary approaches. As an example, in order to understand intracellular protein trafficking (a hot topic in plant biochemistry), Heard et al. (2014) [50] enriched partially overlapping subsamples of endosomal proteomes associated with endomembrane from A. thaliana seedlings and used affinity purification of fluorescent fusion constructs for seven subcellular marker proteins in order to predict its precise or shared location, a strategy that resembles LOPIT [51]

From the current literature it is clear that plant proteomics is moving from 2-DE/MS (still the bases of an important number of works published) to shotgun (bottom up gel free label free). Shotgun will be the dominant platform in the near future if accompanied by a powerful and potent MS analysis and equipment. It is simple and does not require much manipulation of the sample. The iTRAQ, quite popular among the Chinese groups, appeared as the third alternative, even though plant proteomicists did not pay much attention in the past to labelling techniques [52-55]. By using this technique, Pan et al. (2015) [56] have reported up to 13,706 and 12,124 proteins identified with a LTQ-Orbitrap hybrid mass spectrometer in Arabidopsis thaliana roots and leaves. Changes in abundance between plants supplied with different iron concentrations were observed for 886 proteins with only a low percentage of them showing good correlation with transcript levels.

Whatever the employed platform, it is very important that it is validated, as a preliminary step, for the experimental system being analyzed, as for any analytical tool, in order to allow the determination of the number of proteins confidently identified and quantified. Serial dilution of the sample could be enough for validation purposes for those approaches based on label-free [57].

Fourth generation in vitro proteomics, SRM, MRM, or mass-western, sustain hypothesis driven protein analysis. In the surveyed period just one paper, by Rogniaux et al. (2015) [58] was published in Proteomics reporting the use of targeted approach for determining the relative abundance of allergens in several wheat varieties, and showing the potential of the technique for translational, and food traceability, purposes. Although the use of these techniques in plants is still limited, targeted 
proteomics is the way to go for validation of the candidate proteins identified in 2-DE or shotgun analyses. It is certainly a more appropriate follow-up study when compared to qRT-PCR analysis frequently reported in proteomics publications.

Imaging has opened new possibilities for in vivo or in situ proteomics analysis and integration with cellular biology techniques [59], giving, at the same time, the possibility of proteomics analysis at the cellular level [60]. It is a more or less well stablished technique for metabolomics analysis [61] but under development for proteins [62] and plant tissue samples. The technique has been the focus of the Proteomics Tissue Proteomics and Imaging Mass Spectrometry special issue (Vol. 14, Iss. 7-8), in which, two plant studies were reported. Maciel et al. (2014) [24] used the laser ablation imaging technique to evaluate the distribution of selenium and sulfur in leaves of transgenic and nontransgenic A. thaliana. One of the limitations of imaging studies is the processing of high amounts of datasets, and therefore attention has been given to data processing methods, as discussed by Wijetunge et al. [63] and Franceschi and Wehrens (2014) [64].

\section{In silico analysis and data interpretation: software, databases and repositories}

Proteomics, as any -omics approach, generates from hundreds to thousands of identified products, being impossible to analyze and interpret all the data in a classic, mind based, manner [65]. The high amount of data generated and the costs of the analysis are the main reasons for the low number of samples and biological replicates analyzed. Protein profiling requires software and algorithms for protein identification, quantitation, functional and structural characterization, location, interaction, networks, integration, visualization and deposition in databases and repositories. These softwares are continuously created, improved and updated [66-69]. Recently, Sakata and Komatsu (2014) [28] have surveyed and reviewed plant proteomics databases and websites as well as recent technologies related to data collection and annotation.

The use of most algorithms and softwares is far from the current capacity of most plant biochemists working with proteomics. This is the reason why the dry part of the work is in the hands 
of mass spectrometrists or bioinformaticians usually with no expertise in plant biology. This results in, more often than would be advisable, a blind acceptance of the computer provided data without a minimum rationale and critical evaluation of the data confidence, making it difficult to conclude from a biological point of view. The quite dynamic character of the proteomes and the fixed film provided by our experiments makes most interpretations speculative. The situation for a standard plant biochemist is even worse considering the number of algorithms that is continuously appearing [70]. Which one, what for, how to use them and translate to a biological discussion could be hell for most of us $[71,72]$. In this regard, Proteomics a special issue, Proteomics Data Visualization (17, 10). In this and other regular issues a number of reviews discussing these questions have appeared [73-78]

The integration of disseminated data through databases is a pursued objective that will benefit the scientific community. In this direction, The BioMart Community Portal (www.biomart.org) is a good example the plant community should follow [79]. It includes over 800 different biological datasets spanning genomics, proteomics, model organisms, cancer data, ontology information and more.

\section{PROTEOMICS INTEGRATION WITH OTHER -OMICS}

Within the -omics approaches, the advantage, if so, of proteomics, and also metabolomics, is that the information provided is closer to the phenotype [80]. On the other hand, it is much more complicate at the methodological level (i.e. genomics and transcriptomics are favored by PCR and well established microarray techniques). At the molecular level, the phenotype is the result of gene expression through transcriptional and translational events and gene products and metabolites interaction, so proteins are just part of the story. As previously discussed [1], proteomics has limitations, the more relevant being proteome coverage, quantitation, identification in orphan organisms, PTM, and interactomics. Protein identification and quantification cannot always be as confident as we wish because of the organism, experimental design, and the employed techniques and 


\section{7}

278

279

280

281

282

283

284

protocols, making our data interpretation conservative or speculative. In comparative proteomics, the high variability in the protein profile among biological samples and the low number of biological replicates employed in most studies hinder the biological interpretation. Thus, it is not easy to stablish protein abundance ranges in normal (control) samples, as it has been shown by Higdon et al. (2015) [81]. These authors used data from two human proteome studies featured in Nature, and found that protein abundance among biological replicates varied by \pm 4 - to 10 -fold for most proteins, with coefficient of variation (CV) in between 62 and $117 \%$. To control or reduce this $\mathrm{CV}$, it is recommended to compare proteins identified with at least 3 or more unique peptides and normalize abundances against housekeeping proteins with low variability.

Just by itself, and more than generating a list or catalog of protein species, proteomics can provide real biological knowledge; however it would require, first, the use of a model systems, in plants mostly A. thaliana, with a good collection of mutants for reverse and functional genetics studies and, second, an appropriate experimental system, as for example cell suspensions. A good example of this is the paper published by Smith et al. (2015) [82] in which signal transduction pathways mediating plant programmed cell death was studied. By using isobaric-tagged relative and absolute quantitation of proteins present in the extracellular matrix, 33 proteins were identified as putative cell-death regulatory proteins. One of these proteins, the CYCLASE 1, which had previously no known function, was a negative regulator of cell death in Arabidopsis.

The integration of the different-omics, in the direction of systems biology, is far from being a reality or was only possible for a reduced number of research groups or through large international consortia [83]. Systems biology, wide genomic approaches, and the recently coined proteogenomics, in combination with image phenotyping, classical molecular, physiological and cell biology approaches $[84,85]$ will get us to a deeper and better rather than to a partial and biased knowledge of the biological systems. This knowledge is highly relevant for crop improvement aiming at productivity yield increases and survival under extreme environmental conditions through classical, molecular marker assisted, and biotechnological breeding [11, 86-88] 

response to waterlogging and resulted, in general, in good correlations between both data [54]. But this is not necessarily the rule and it depends on the gene or gene groups, being the consequence of transcriptional, posttranscriptional, translational and posttranslational events. Abundance of both molecules depends not only on their synthesis but also on their stability and degradation. It is also known that different mRNAs possess different translational efficacy [89].

At least one paper, by Gupta et al. (2015) [90], has been published in which integrated proteomics and metabolomics have been employed as experimental approaches. They analyzed differences between soybean lines with different color in the seed coat. Differences in flavonoids, which provide the molecular basis of the color, were correlated with levels in pathway-related enzymes. Finally, there are not many papers in which the combined use of the three -omics approaches are reported. In the surveyed period, two papers dealt with the detection of unintended effects in transgenic maize $[91,92]$.

In any case, single and easy multi-omics approach experiments can be always performed and the preliminary steps of the workflow should be evaluated as one of the limitations is just preparative. In order to make confident data comparison or validation, these preparations should come from the same sample. Independent experiments or extractions can originate artefacts or biases, mostly coming from the inherent biological variability. In this direction, recent workflows aimed to extract biomolecules (DNA, RNAs, proteins, and metabolites) have been developed [93]. Despite dedicated workflows showed their efficiency, combined protocols demonstrated their performance in isolating biomolecules suitable to be processed by high-throughput pipelines (gel free label free proteomics, high-throughput metabolomics analyses and next generation sequencing transcriptomic analyses of messenger or regulatory RNAs) while avoiding biases related to the use of not exactly the same sample for the different -omics level analyses. The full overlapping of the datasets makes it easier to interpret and analyze the data when performing a Systems Biology-based integrative approach. 
Proteogenomics is a relatively new and emerging field at the intersection of genomics and proteomics, being part of the wide genomics approaches. Although it may have multiple derivations (RNA and protein correlation studies, proteomics and data validation, gene sequence analysis, among others), in the simplest way it can be understood as the use of proteomics data and protein MS derived

316 sequences to improve gene sequence annotations [94-96]. From the proteomics perspective it has an important feedback as correct sequence annotation will increase the number and confidence of the protein identification [97], favoring, at the same time the studies of non-model organisms [98]. It will also go deeper into the knowledge of posttranscriptional and translational events giving rise to the different gene products, protein species or proteoforms [99].

The topic has been deeply reviewed in Proteomics (Proteogenomics special issue; Vol. 14, Iss. 23-24). Proteogenomic experiments and prediction tools will differ between genome-sequenced and-unsequenced, as well as eukaryotic or prokaryotic organisms $[95,100]$.

\section{FROM PROTEOMICS TO BIOLOGY}

Cell communication, receptors, and signaling pathways, are good examples of how proteomics can contribute to biological knowledge and be used for translational purposes. This topic has been well presented in a recent, excellent review, by Walton et al. (2015) [27]. Signaling is a hot topic of great relevance in biology mainly approached by using functional and reverse genetics. It is quite challenging as it deals with minor and recalcitrant, membrane bound, proteins, PTMs and interactions, whose investigation requires integration with classic, transcriptomics and metabolomics approaches. In the recent literature surveyed in the reviewed period, this topic is well covered with both review papers and original contributions. Some other topics are also of interest but are somehow related to this or are less represented in the most recent literature. This is the case of plant developmental processes, plant-pathogen interactions and responses to other biotic stresses [101-104].

Plants chemically communicate and interact with their biotic and abiotic surroundings, responding and adapting its biology to it. They also complete their developmental program (from seed 
germination to seed production through vegetative and reproductive phases) in coordination with seasonal changes noticed mainly as variations in light, temperature and water availability. There are also daily changes associated to a circadian clock timed by, among other factors, light regimes. The integration of environmental responses with development is crucial for plant survival, growth, and from an agronomic point of view, productivity. Plants, as sessile organisms, are very versatile, and their genome expression and metabolism is continuously reprogrammed in response to physicochemical and biological stimuli. Despite the high number of specialized organs, tissues and cell types within a plant, all of them respond coordinately, implicating in good intercellular connexion systems with chemical messages between receptor and effector cells. At the individual cell level, gene expression changes are mediated by receptors, intracellular and interorganule signals, and signal transduction pathways resulting in changes in gene expression through transcriptional, translational and posttranslational events. Quite a number of molecules are known that act as external or internal chemical signals, including phytohormones, and those from microorganisms (symbionts, parasites or pathogens) [101, 103-106]. On the other side, receptors and signal transduction pathways and their components are starting to be characterized [31, 107].

Proteomics is contributing to the above processes through descriptive, comparative, PTMs, and interaction studies. Walton et al. (2015) [26] updated the contribution in these areas and the different strategies employed in dissecting plant hormone signaling. Some following papers dealing with PTMs and interactomics related to cell signaling are highlighted. Others, using descriptive or comparative proteomics approaches have been skipped from this review.

Protein phosphorylation is the most common mechanism of signal and because of that phosphoproteomics is the immediate strategy to be employed. Silva-Sanchez et al., (2015) [108], and Li et al., (2015) [29] have summarized the current methodologies used in extracting, enriching, and fractionating phosphoproteins, and in their MS analyses for identification, quantitation, and phosphorylation site mapping, conclude on cross-talk between different PTMs (phosphorylation, glycosylation, and redox modifications) and data validation. They provided a list of phosphoproteins, 
softwares and databases. As an alternative to the current protocols, Han et al. (2015) [109] have proposed a faster workflow analysis in the case of sample amount limitation that increases coverage; it included integrated multistep enzymatic digestion, phosphopeptide enrichment and database search (iMEED). In an attempt to elucidate post-translational processes mediating circadian events,

Choudhary et al. (2015) [110] performed a phosphoprotein analysis employing TiO2 phosphopeptide and MS analysis. They identified 1586 phosphopeptides on 1080 protein groups, out of which 102 changed in abundance, including clock proteins (ELF4, PRR3), transcription factors and kinases. By using isotope-assisted quantitative phosphoproteomics, Minkoff et al. (2015) [111] carried out a comparative phosphoproteomics study between wild and mutant ABA-insensitive A. thaliana plants. Multiple changes occurred as early as 5 minutes after ABA treatment, with a reduction of the phosphorylation events in the mutant.

Reactive oxygen species overproduction is a typical plant response to oxidative stress, these species acting as signals and causing protein redox state changes [30,112, 113]. The reversible PTM methionine oxidation was studied in A. thaliana plants by Jacques et al. (2015) [114] using wild and catalase-2 knock-out mutants. Over 500 sites of oxidation in 400 proteins were reported. They showed that the activity of two specific glutathione-S-transferases was significantly reduced upon oxidation. Konert et al. (2015) [115] have gone deeper into the mechanism of ROS signaling and metabolic adjustment in A. thaliana. By using genetic, biochemical, and a combination of MS data-dependent acquisition and SRM, they showed how protein phosphatase 2A, through its regulatory $\mathrm{B}^{\prime} \gamma$ subunit, interact with a cytosolic aconitase and control mitochondrial alternative oxidases.

Interactomics is the most challenging and less developed proteomics approach, but the most concluding one in order to probe in vitro and in situ better than in silico interactions between proteins and proteins and DNA, RNA or metabolites. It will help in developing models from networks mediating receptor capture of signals and gene expression changes. The standard proteomics analysis involves the capture of protein partners (affinity purification or immunoprecipitation) coupled to MS analysis. Other published approaches included in vivo cross-linking with para-formaldehyde and 
immunoaffinity purification of cross-linked protein. It has been used by Obermeyer et al., (2014) [116] in the search for $\mathrm{H}(+)$ ATPase complexes in lily pollen. These techniques require high amount of sample and involve excessive manipulation, being subjected to biases or false positives. Microarray technology will be, with no doubt, the future for more feasible experiments in which dynamic interactions could be observed through time course experiments [117]. As an alternative, the parallel analysis of genomic, transcriptomics, and proteomics experiments could be performed [118].

\section{Some missed relevant papers}

Last but not least in the surveyed period are the publications covering topics that deserve to be mentioned in a general plant proteomics review, either because they report on technical innovations or novel applications to the plant biology research. As we did not intend to be exhaustive, and due to space limitation, we will just mention them.

Such is the case of the top-down proteomics [45, 119-121], N-acetylome [122], degradation of proteins with $\mathrm{N}$-termini containing basic amino acids (the Arg/N-end rule) [25], and single cell type proteomics analysis. Plant organs are complex in terms of the different types of tissues and cells that are formed, with each one having its own proteome signature. Thus, for comparative proteomics purposes it is possible that only a discrete number of cells respond to the experimental variable under study and if so, by extracting the whole organ we might be diluting the response. In order to decipher single proteome analysis new methodologies should be developed [86]. Up to now the laser microdissection [123] has been applied, however it has been of very limited use with plants. What is close to be a technical reality for transcriptomics looks impossible for proteomics [124].

\section{Acknowledgements}

Thanks to the University of Córdoba (Programas Propio y de Fortalecimiento) and CNPq (AM, and JVJN) for financial support. L.V. was generously funded by the Juan de la Cierva Programme (JCI2012-12444, Spanish Ministry of Economy and Competitiveness). Rosa Sánchez Lucas is a recipient 
of a Formación de Profesorado Universitario fellowship (FPU14/00186, Spanish Ministry of

416 Education, Culture and Sport). The funders had no role in the study design, data collection and

417 analysis, decision to publish, or preparation of the manuscript.

\section{References}

419 [1] Jorrin-Novo, J.V., Pascual, J., Sanchez-Lucas, R., Romero-Rodriguez, M.C., et al., Fourteen years of plant proteomics reflected in Proteomics: Moving from model species and 2DE-based approaches to orphan species and gel-free platforms. Proteomics 2015, 5-6, 1089-1112.

[2] Deswal, R., Abat, J.K., Sehrawat, A., Gupta, R., et al., First systematic plant proteomics workshop in Botany Department, University of Delhi: Transferring proteomics knowledge to next-generation researchers and students. Proteomics 2014, 14, 1581-1586.

[3] Dunn, M.J., Proteomics into its third decade. Proteomics 2015, 15, 1-2.

[4] Thongboonkerd, V., LaBaer, J., Domont, G.B., Recent advances of proteomics applied to human diseases. J. Proteome Res., 2014, 13, 4493-4496.

428 [5] Kennedy, D., Building agricultural research. Science 2014, 346, 13.

429 [6] Lüthje, S., Deswal, R., Agrawal, G.K., Editorial. Plant-based foods: seed, nutrition and human 430 health. Proteomics 2015, 15, 1638.

431 [7] Gregorich, Z.R., Ge, Y., Top-down proteomics in health and disease: challenges and opportunities. 432 Proteomics 2014, 14, 1195-1210.

433 [8] Ebhardt, H.A., Root, A., Sander, C., Aebersold, R., Applications of targeted proteomics in systems 434 biology and translational medicine. Proteomics 2015 15, 3193-3208.

[9] FAO., Globally almost 870 million chronically undernourished - new hunger report. Food and Agriculture Organization of the United Nations, Rome 2012. Report at: http://www.fao.org/news/story/en/item/161819/icode/.

438 [10] Gutiérrez, R.A., Green, P.J., Keegstra, K., Ohlrogge, J.B., Phylogenetic profiling of the Arabidopsis thaliana proteome: what proteins distinguish plants from other organisms? Genome Biol. 2004, R53. 
441 [11] Rodríguez, R.M., López-Vázquez, A., López-Larrea, C., Immune systems evolution. Adv. Exp.

442 Med. Biol. 2012, 739, 237-251.

443 [12] Suh, L.S., Suh, S.J., Sokol, P.A., Ohman, D.E., A simple alfalfa seedling infection model for

444 Pseudomonas aeruginosa strains associated with cystic fibrosis shows AlgT (sigma-22) and RhlR 445 contribute to pathogenesis. Proc. Natl. Acad. Sci. 2002, 99, 15699-15704.

446 [13] Weidle, U.H., Tiefenthaler, G., Schiller, C., Weiss, E.H., et al., Prospects of bacterial and plant 447 protein-based immunotoxins for treatment of cancer. Cancer Genom. Proteom. 2014, 11, 25-38.

448 [14] Leung, P.S., Shu, S.A., Chang, C., The changing geoepidemiology of food allergies. Clin. Rev. 449 Allergy Immunol. 2014, 46, 169-179.

450 [15] Halim, A., Carlsson, M.C., Madsen, C.B., Brand, S., et al., Glycoproteomic analysis of seven 451 major allergenic proteins reveals novel post-translational modifications. Mol. Cell Proteomics 2015, $45214,191-204$.

453 [16] Heidstra, R., Sabatini, S., Plant and animal stem cells: similar yet different. Nat. Rev. Mol. Cell 454 Biol. 2014, 15, 301-312.

455 [17] McKnight, T.D., Riha, K., Shippen, D.E., Telomeres, telomerase, and stability of the plant genome. Plant Mol. Biol. 2002, 48, 331-337.

[18] Uhlén, M., Fagerberg, Hallström, B.M., Lindskog, C., et al., Proteomics. Tissue-based map of the 458 human proteome. Science 2015, 347, 1260419.

[19] Wilhelm, M., Schlegl, J., Hahne, H., Moghaddas Gholami, A., et al., Mass-spectrometry-based draft of the human proteome. Nature 2014, 509, 582-587.

461 [20] Sharma, T.K., Ramanathan, R., Rakwal, R., Agrawal, G.K, Bansal, V., Moving forward in plant 462 food safety and security through NanoBioSensors: Adopt or adapt biomedical technologies? 463 Proteomics 2015, 15, 1680-1692.

464 [21] Fang, X.P., Chen, J.P., Dai, L.Y., Ma, H.S., et al., Proteomic dissection of plant responses to 465 various pathogens. Proteomics 2015, 15, 1525-1543. 
[22] Ngara, R., Ndimba, B., Understanding the complex nature of salinity and drought-stress response

467 in cereals using proteomics technologies. Proteomics 2014, 14, 611-621.

[23] Kim, S.T., Kim, S.G., Agrawal, G.K., Kikuchi, S., Rakwal, R., Rice proteomics: A model system for crop improvement and food security. Proteomics 2014, 14, 593-610.

[24] Maciel, B.C.M., Barbosa, H.S., Pessôa, G.S., Salazar, M.M., et al., Comparative proteomics and metallomics studies in Arabidopsis thaliana leaf tissues: Evaluation of the selenium addition in transgenic and nontransgenic plants using two-dimensional difference gel electrophoresis and laser ablation imaging. Proteomics 2014, 14, 904-912.

[25] Zhang, H., Deery, M.J., Gannon, L., Powers, S.J. e al., Quantitative proteomics analysis of the 475 Arg/N-end rule pathway of targeted degradation in Arabidopsis roots. Proteomics 2015, 15, 24474762457.

477 [26] Venne, A.S., Solari, F.A., Faden, F., Paretti, T., et al., An improved workflow for quantitative N478 terminal charge-based fractional diagonal chromatography (ChaFRADIC) to study proteolytic events 479 in Arabidopsis thaliana. Proteomics 2015, 15, 2458-2469.

480 [27] Walton, A., Stes, E., De Smet, I., Goormachtig, S., Gevaert, K., Plant hormone signaling through 481 the eye of the mass spectrometer. Proteomics 2015, 15, 1113-1126.

482 [28] Sakata, K., Komatsu, S., Plant proteomics: from genome sequencing to proteome databases and 483 repositories. Methods Mol Biol. 2014, 1072, $29-42$.

[29] Li, J., Silva-Sanchez, C., Zhang, T., Chen, S., Li, H., Phosphoproteomics technologies and 485 applications in plant biology research. Front Plant Sci. 2015, 6, 430.

486 [30] Akter, S., Huang, J., Waszczak, C., Jacques, S., et al., Cysteines under ROS attack in plants: a 487 proteomics view. J Exp Bot. 2015, 66, 2935-2944. 
[31] Tripathi, P., Rabara, R.C., Rushton, P.J.N., A systems biology perspective on the role of WRKY transcription factors in drought responses in plants. Planta. 2014. 239, 255-266.

[32] Jorrin-Novo, J., 2015. Scientific standards and MIAPEs in Plant Proteomics research and publications: do we follow them? Front. Plant Sci. 2015; 6, 473.

[33] Lesur, A., Domon, B., Advances in high-resolution accurate mass spectrometry application to targeted proteomics. Proteomics 2015, 15, 880-890.

[34] Lee, D.C.H., Jones, A.R., Hubbard, S.J., Computational phosphoproteomics: From identification to localization. Proteomics 2015, 15, 950-963.

[35] Wu, X.L., Gong, F.P., Wang, W., Protein extraction from plant tissues for 2DE and its application in proteomic analysis. Proteomics 2014, 14, 645-658.

[36] Lee, H.M., Gupta, R., Kim, S.H., Wang, Y., et al., Abundant storage protein depletion from tuber proteins using ethanol precipitation method: Suitability to proteomics study. Proteomics 2015, 15, 1765-1769.

501 [37] Kim, Y.J., Wang, Y., Gupta, R., Kim, S.W., et al., Protamine sulfate precipitation method 502 depletes abundant plant seed-storage proteins: A case study on legume plants. Proteomics 2015, 15, $503 \quad 1760-1764$.

504 [38] Wildgruber, R., Weber, G., Wise, P., Grimm, D., Bauer, J., Free-flow electrophoresis in 505 proteome sample preparation. Proteomics 2014, 14, 629-636.

506 [39] Moritz, C.P., Marz, S.X., Reiss, R., Schulenborg, T., Friauf, E., Epicocconone staining: A 507 powerful loading control for Western blots. Proteomics 2014, 14, 162-168. 
510 [41] Hildonen, S., Halvorsen, T.G., Reubsaet, L., Why less is more when generating tryptic peptides

511 in bottom-up proteomics. Proteomics 2014, 14, 2031-2041.

512 [42] George, I.S., Fennell, A.Y., Haynes, P.A., Protein identification and quantification from 513 riverbank grape, Vitis riparia: Comparing SDS-PAGE and FASP-GPF techniques for shotgun 514 proteomic analysis. Proteomics 2015, 15, 3061-3065.

515 [43] Valledor, L., Weckwerth, W. In: Jorrin-Novo, J., Weckwerth, W., Wienkoop, S., Komatsu, S., 516 (Eds) Plant Proteomics: Methods and Protocols. Springer. Germany. pp 347-358.

[44] Cannon, J.R., Kluwe, C., Ellington, A., Brodbelt, J.S., Characterization of green fluorescent proteins by $193 \mathrm{~nm}$ ultraviolet photodissociation mass spectrometry. Proteomics 2014, 14, 1165-1173.

519

520

521

522

[45] Dang, X.B., Young, N.L., Ultraviolet photodissociation enhances top-down mass spectrometry as demonstrated on green fluorescent protein variants. Proteomics 2014, 14, 1128-1129.

[46] Valledor, L., Jorrín, J., Back to the basics: Maximizing the information obtained by quantitative two dimensional gel electrophoresis analyses by an appropriate experimental design and statistical analyses. J. Proteomics 2011, 74, 1-18.

[47] Krahmer, J., Hindle, M.M., Martin, S.F., Le Bihan, T., Millar, A.J., Sample preparation for phosphoproteomic analysis of circadian time series in Arabidopsis thaliana. Methods Enzymol. 2015, $551,405-431$.

[48] Trotschel, C., Poetsch, A., Current approaches and challenges in targeted absolute quantification of membrane proteins. Proteomics 2015, 15, 915-929.

[49] Clarke, V.C., Loughlin, P.C., Gavrin, A., Chen, C., et al., Proteomic analysis of the soybean symbiosome identifies new symbiotic proteins. Mol Cell Proteomics 2015, 14, 1301-1322. 
531 [50] Heard, W., Sklenář, J., Tomé, D.F., Robatzek, S., Jones, A.M., Identification of Regulatory and

532 Cargo Proteins of Endosomal and Secretory Pathways in Arabidopsis thaliana by Proteomic

533 Dissection. Mol. Cell Proteomics 2015, 14, 1796-1813.

534 [51] Dunkley, T.P.J., Watson, R., Griffin, J.L., Dupree, P., Lilley, K.S., Localization of Organelle 535 Proteins by Isotope Tagging (LOPIT). Mol. Cell Proteomics 2004, 3, 1128-1134.

536 [52] Glibert, P., Van Steendam, K., Dhaenens, M., Deforce, D., iTRAQ as a method for optimization: 537 Enhancing peptide recovery after gel fractionation. Proteomics 2014, 14, 680-684.

[53] Ma, S., Sun, Y., Zhao, X., Xu, P., Recent advance in high accuracy iTRAQ for quantitative proteomics [Article in Chinese]. Sheng Wu Gong Cheng Xue Bao 2015, 30, 1073-1082.

[54] Yu, F., Han, X.S., Geng, C.J., Zhao, Y.X., et al., Comparative proteomic analysis revealing the complex network associated with waterlogging stress in maize (Zea mays L.) seedling root cells. Proteomics 2015, 15, 135-147.

[55] Zargar, S.M., Kurata, R., Inaba, S., Oikawa, A., et al., Quantitative proteomics of Arabidopsis shoot microsomal proteins reveals a cross-talk between excess zinc and iron deficiency, Proteomics $5452015,15,1196-1201$.

546 [56] Pan, I.C., Tsai, H.H., Cheng, Y.T., Wen, T.N., et al., Post-Transcriptional Coordination of the 547 Arabidopsis Iron Deficiency Response is Partially Dependent on the E3 Ligases RGLG1 and RGLG2. 548 Mol Cell Proteomics 2015, 14, 2733-2752.

[57] González-Fernández, R., Aloria, K., Arizmendi, J.M., Jorrin-Novo, J.V., Application of Label550 Free shotgun nUPLC-MS ${ }^{\mathrm{E}}$ and 2-DE approaches in the study of Botrytis cinerea mycelium. $J$. $551 \quad$ Proteome Res 2013, 12, 3042-3056. 
[58] Rogniaux, H., Pavlovic, M., Lupi, R., Lollier, V., et al., Allergen relative abundance in several wheat varieties as revealed via a targeted quantitative approach using MS. Proteomics 2015, 15, 1736-

5541745.

555 [59] Caprioli, R.M., Imaging mass spectrometry: Molecular microscopy for enabling a new age of 556 discovery. Proteomics 2015, 15, 807-809.

557 [60] Li, H., Smith, B.K., Shrestha, B., Márk, L., Vertes, A., Automated cell-by-cell tissue imaging and single-cell analysis for targeted morphologies by laser ablation electrospray ionization mass spectrometry. Methods Mol. Biol. 2015, 1203, 117-127.

[61] Bartels, B., Svatoš, A., Spatially resolved in vivo plant metabolomics by laser ablation-based 561 mass spectrometry imaging (MSI) techniques: LDI-MSI and LAESI. Front Plant Sci. 2015, 6, 471.

562 [62] Kiss, A., Smith, D.F., Reschke, B.R., Powell, M.J., Heeren, R.M.A., Top-down mass spectrometry imaging of intact proteins by laser ablation ESIFT-ICR MS. Proteomics 2014, 14, 1283 5641289.

565 [63] Wijetunge, C.D., Saeed, I., Boughton, B.A., Spraggins, J.M., et al., EXIMS: an improved data 566 analysis pipeline based on a new peak picking method for EXploring Imaging Mass Spectrometry 567 data. Bioinformatics 2015 31, 3198-3206.

568 [64] Franceschi, P., Wehrens, R., Self-organizing maps: A versatile tool for the automatic analysis of 569 untargeted imaging datasets. Proteomics 2014, 14, 853-861.

570 [65] Rabilloud, T., Lescuyer, P., The proteomic to biology inference, a frequently overlooked concern 571 in the interpretation of proteomic data: A plea for functional validation. Proteomics 2014, 14, 157572 161.

573 [66] Fellers, R.T., Greer, J.B., Early, B.P., Yu, X., et al., ProSight Lite: graphical software to analyze 574 top-down mass spectrometry data. Proteomics 2015, 15, 1235-1238. 
[67] Qi, D., Lawless, C., Teleman, J., Levander, F., et al., Representation of selected-reaction monitoring data in the mzQuantML data standard. Proteomics 2015, 15, 2592-2596.

[68] San Clemente, H., Jamet, E., WallProtDB, a database resource for plant cell wall proteomics. Plant Methods 2015, 11, 2.

579 [69] Wen, B., Du, C., Li, G., Ghali, F., et al., IPeak: An open source tool to combine results from multiple MS/MS search engines. Proteomics 2015, 15, 2916-2920.

[70] Gallardo, O., Ovelleiro, D., Gay, M., Carrascal, M., Abian, J., A collection of open source applications for mass spectrometry data mining. Proteomics 2014, 14, 2275-2279.

583 [71] Oveland, E., Muth, T., Rapp, E., Martens, L., et al., Viewing the proteome: How to visualize

584

585

586

587

588 proteomics data? Proteomics 2015, 15, 1341-1355.

[72] Bilbao, A., Varesio, E., Luban, J., Strambio-De-Castillia, C., et al., Processing strategies and software solutions for data-independent acquisition in mass spectrometry. Proteomics 2015, 15, 964980.

[73] Laukens, K., Naulaerts, S., Vanden Berghe, W., Bioinformatics approaches for the functional interpretation of protein lists: From ontology term enrichment to network analysis. Proteomics 2015, 15, 981-996.

[74] Mikalsen, S.O., Proteomics made more accessible. Proteomics 2014, 14, 980-990.

[75] Vaudel, M., Venne, A.S., Berven, F.S., Zahedi, R.P., et al., Shedding light on black boxes in protein identification. Proteomics 2014, 14, 1001-1005.

[76] Aiche, S., Sachsenberg, T., Kenar, E., Walzer, M., et al., Workflows for automated downstream data analysis and visualization in large-scale computational mass spectrometry. Proteomics 2015, 15, 1443-1447. 
597 [77] Perez-Riverol, Y., Alpi, E., Wang, R., Hermjakob, H., Vizcaino, J.A., Making proteomics data 598 accessible and reusable: Current state of proteomics databases and repositories. Proteomics 2015, 15, $599 \quad 930-949$.

600 [78] Tyanova, S., Temu, T., Carlson, A., Sinitcyn, P., et al., Visualization of LC-MS/MS proteomics 601 data in MaxQuant. Proteomics 2015, 15, 1453-1456.

[79] Smedley D, Haider S, Durinck S, Pandini L, et al., The BioMart community portal: an innovative

603 alternative to large, centralized data repositories. Nucleic Acids Res 2015, 43(W1), W589-598.

[80] Feussner, I., Polle, A., What the transcriptome does not tell - proteomics and metabolomics are closer to the plants' patho-phenotype, Curr Opin Plant Biol. 2015, 26, 26-31.

[81] Higdon, R., Kolker, E., Can "normal" protein expression ranges be estimated with highthroughput proteomics? J Proteome Res., 2015, 14, 2398-2407.

[82] Smith, S.J., Kroon, J.T., Simon, W.J., Slabas, A.R., et al., A Novel Function for Arabidopsis CYCLASE1 in Programmed Cell Death Revealed by Isobaric Tags for Relative and Absolute Quantitation (iTRAQ) Analysis of Extracellular Matrix Proteins, Mol Cell Proteomics 2015, 14, $1556-1568$.

[83] Hussein, S.M., Puri, M.C., Tonge, P.D., Benevento, M., et al., Genome-wide characterization of the routes to pluripotency. Nature 2014, 516, 198-206.

[84] Fraas, S., Lüthen, H., Novel imaging-based phenotyping strategies for dissecting crosstalk in plant development, J. Exp. Bot. 2015, 66, 4947-2955.

[85] Stitt, M., Gibon, Y., Why measure enzyme activities in the era of systems biology? Trends Plant Sci., 2014, 19, 256-265.

[86] Misra, B.B., Assmann, S.M., Chen S., Plant single-cell and single-cell-type metabolomics. Trends Plant Sci. 2014, 19, 637-646. 
621 for crop improvement. Crit Rev Biotechnol. 2015, 14, 1-14.

[88] Zivy, M., Wienkoop, S., Renaut, J., Pinheiro, C., et al., The quest for tolerant varieties: the importance of integrating "omics" techniques to phenotyping. Front Plant Sci. 2015, 6, 448.

[89] Browning, K.S., Bailey-Serres, J., Mechanism of cytoplasmic mRNA translation. Arabidopsis Book 2015, 13, e0176.

[90] Gupta, R., Min, C.W., Kim, S.W., Wang, Y., et al., Comparative investigation of seed coats of brown- versus yellow-colored soybean seeds using an integrated proteomics and metabolomics approach. Proteomics 2015, 15, 1706-1716.

[91] Barros, E., Lezar, S., Anttonen, M.J., Van Dijk, J.P., Comparison of two GM maize varieties with a near-isogenic non-GM variety using transcriptomics, proteomics and metabolomics. Plant Biotechnol J. 2010, 8, 436-451.

[92] Decourcelle, M., Perez-Fons, L., Baulande, S., et al., Combined transcript, proteome, and metabolite analysis of transgenic maize seeds engineered for enhanced carotenoid synthesis reveals 634 pleotropic effects in core metabolism. J. Exp. Bot. 2015, 66, 3141-3150.

635 [93] Valledor, L., Escandon, M., Meijon, M., Nukarinen, E., et al., A universal protocol for the 636 combined isolation of metabolites, DNA, long RNAs, small RNAs, and proteins from plants and 637 microorganisms. Plant J. 2014; 79, 173-180.

638

639

640

[94] Gupta, N., Tanner, S., Jaitly, N., Adkins, J.N., et al. Whole proteome analysis of posttranslational modifications: Applications of mass-spectrometry for proteogenomic annotation. Genome Res. 2007, 17, 1362-1377.

641

[95] Kucharova, V., Wiker, H.G., Proteogenomics in microbiology: Taking the right turn at the 642 junction of genomics and proteomics. Proteomics 2015, 14, 2660-2675. 
[96] Wang, X.J., Liu, Q., Zhang, B., Leveraging the complementary nature of RNA-Seq and shotgun proteomics data. Proteomics 2014, 14, 2676-2687.

[97] Romero-Rodríguez, C., Pascual, J., Valledor, L., Jorrín-Novo, J.V., Improving the quality of protein identification in non-model species. Characterization of Quercus ilex seed and Pinus radiata needle proteomes by using SEQUEST and custom databases. J. Proteomics 2014, 105, 85-91.

[98] Armengaud, J., Trapp, J., Pible, O., Geffard, O., et al.,. Non-model organisms, a species endangered by proteogenomics. J. Proteomics 2014, 105, 5-18.

[99] Gawron, D., Gevaert, K., Van Damme, P., The proteome under translational control. Proteomics $2015,14,2647-2659$.

652 [100] Chapman, B., Bellgard, M., High-throughput parallel proteogenomics: A bacterial case study. Proteomics 2014, 14, 2780-2789.

654 [101] De-la-Peña, C., Loyola-Vargas, V.M., Biotic interactions in the rhizosphere: a diverse cooperative enterprise for plant productivity. Plant Physiol. 2014, 166, 701-719.

[102] Gupta, R., Lee, S.E., Agrawal, G.K., Rakwal, R., et al., Understanding the plant-pathogen interactions in the context of proteomics-generated apoplastic proteins inventory. Front Plant Sci. 2015, 6, 352. [103] Hurley, B., Subramaniam, R., Guttman, D.S., Desveaux, D., Proteomics of 659 effector-triggered immunity (ETI) in plants. Virulence 2014, 5, 752-760.

[104] Zdarska, M., Dobisová, T., Gelová, Z., Pernisová, M., et al., Illuminating light, cytokinin, and ethylene signalling crosstalk in plant development. J Exp Bot. 2015, 66, 4913-4931.

[105] Heuberger, A.L., Robison, F.M., Lyons, S.M., Broeckling, C.D., Prenni, J.E., Evaluating plant immunity using mass spectrometry-based metabolomics workflows. Front Plant Sci. 2014, 5, 291.

664

[106] Walley, J., Xiao, Y., Wang, J.Z., Baidoo, No E.EPlastid-produced interorgannellar stress signal 665 MEcPP potentiates induction of the unfolded protein response in endoplasmic reticulum. Proc Natl 666 Acad Sci USA. 2015, 112, 6212-6217. 
667 [107] Grones, P., Griml, J., Auxin transporters and binding proteins at a glance. J Cell Sci. 2015, 128, $668 \quad 1-7$.

669 [108] Silva-Sanchez, C., Li, H.Y., Chen, S.X., Recent advances and challenges in plant 670 phosphoproteomics. Proteomics 2015, 15, 1127-1141.

671 [109] Han, D., Jin, J., Yu, J., Kim, K., Kim, Y., Integrated approach using multistep enzyme 672 digestion, $\mathrm{TiO} 2$ enrichment, and database search for in-depth phosphoproteomic profiling. Proteomics $6732015,15,618-623$.

[110] Choudhary, M.K., Nomura, Y., Wang, L., Nakagami, H., Somers, D.E. Quantitative Circadian Phosphoproteomic Analysis of Arabidopsis Reveals Extensive Clock Control of Key Components in 676 Physiological, Metabolic, and Signaling Pathways. Mol Cell Proteomics 2015, 14, 2243-2260.

[111] Minkoff, B.B., Stecker, K.E., Sussman, M.R., Rapid Phosphoproteomic Effects of Abscisic Acid (ABA) on Wild-Type and ABA Receptor-Deficient A. thaliana Mutants. Mol Cell Proteomics $2015,14,1169-1182$.

[112] Liu, P., Zhang, H.M., Wang, H., Xia, Y.J., Identification of redox-sensitive cysteines in the 681 Arabidopsis proteome using OxiTRAQ, a quantitative redox proteomics method. Proteomics 2014, $682 \quad 14,750-762$.

683 [113] Dietz, K.J., Redox regulation of transcription factors in plant stress acclimation and 684 development. Antioxid Redox Signal. 2014, 21, 1356-1372.

685 [114] Jacques, S., Ghesquière, B., De Bock, P.J., Demol, H., et al., Protein Methionine Sulfoxide 686 Dynamics in Arabidopsis thaliana under Oxidative Stress. Mol Cell Proteomics 2015, 14, 1217-1229.

687 [115] Konert, G., Trotta, A., Kouvonen, P., Rahikainen, M., et al., Protein phosphatase 2A (PP2A) 688 regulatory subunit $\mathrm{B}^{\prime} \gamma$ interacts with cytoplasmic ACONITASE 3 and modulates the abundance of 689 AOX1A and AOX1D in Arabidopsis thaliana. New Phytol. 2015, 205, 1250-1263. 
690 [116] Pertl-Obermeyer, H., Schulze, W.X., Obermeyer, G., In vivo cross-linking combined with mass 691 spectrometry analysis reveals receptor-like kinases and $\mathrm{Ca}(2+)$ signalling proteins as putative 692 interaction partners of pollen plasma membrane H(+) ATPases. J Proteomics 2014, 108, 17-29.

693 [117] Wang, H., Li, H., Zhang, W., Wei, L.M., et al., Multiplex profiling of glycoproteins using a 694 novel bead-based lectin array. Proteomics 2014, 14, 78-86.

695 [118] Wang, J.X., Peng, X.Q., Peng, W., Wu, F.X., Dynamic protein interaction network construction 696 and applications. Proteomics 2014, 14, 338-352.

[119] Dang, X.B., Scotcher, J., Wu, S., Chu, R.K., et al,. The first pilot project of the consortium for top-down proteomics: A status report. Proteomics 2014, 14, 1130-1140.

[120] Erba, E.B., Investigating macromolecular complexes using top-down mass spectrometry. Proteomics 2014, 14, 1259-1270.

[121] Gault, J., Malosse, C., Machata, S., Millien, C., et al., Complete posttranslational modification mapping of pathogenic Neisseria meningitidis pilins requires top-down mass spectrometry. Proteomics 20214, 14, 1141-1151.

704 [122] Dinh, T.V., Bienvenut, W.V., Linster, E., Feldman-Salit, A., et al., Molecular identification and 705 functional characterization of the first $\mathrm{N} \alpha$-acetyltransferase in plastids by global acetylome profiling. 706 Proteomics 2015, 15, 2426-2435.

[123] Gautam, V., Sarkar, A.K., Laser assisted microdissection, an efficient technique to understand tissue specific gene expression patterns and functional genomics in plants. Mol Biotechnol. 2015, 57, 299-308. 